incident AKI. Baseline AKI was associated with increased D90 mortality compared to patients without baseline or incident AKI. Incident AKI was associated with the highest mortality (Figure 1). There was no difference in mortality between patients with a baseline AKI that resolved by D7 or persisted (Breslow Chi-square 0.227, $\mathrm{p}=0.633$ ). Patients with incident AKI had significantly higher bilirubin (mean $374 \mathrm{mmol} / \mathrm{L}$ vs $281, \mathrm{p}<0.001)$, INR (2.0 vs $1.8, \mathrm{p}=0.001)$, and neutrophil count (8.1 vs $7.1, \mathrm{p}=0.031$ ) than those without baseline or incident AKI. Prednisolone treatment was associated with a reduced risk of incident AKI (odds ratio 0.53, 95\% confidence interval $0.34-0.81, \mathrm{p}=0.003)$. Age, gender, baseline observations and hepatic encephalopathy were not associated with incident AKI.

Conclusions Incident AKI at D7 confers a worse prognosis than either no or baseline AKI. This highlights the need for proactive monitoring and treatment of factors predisposing to AKI in patients with $\mathrm{AH}$, particularly for patients with markers of severe disease.

\section{Gastroenterology service}

\section{ATU-4 E-HEALTH VERSUS STANDARD CARE IN INFLAMMATORY BOWEL DISEASE MANAGEMENT: A SYSTEMATIC REVIEW AND META-ANALYSIS}

\begin{abstract}
${ }^{1}$ Anish John Kuriakose Kuzhiyanjal* ${ }^{*}{ }^{1}$ Gaurav Nigam, ${ }^{2}$ Georgios Antoniou, ${ }^{3}$ Raymond Cross, ${ }^{4}$ Francis Farraye, ${ }^{5}$ Jimmy Limdi. ${ }^{1}$ The Pennine Acute Hospitals NHS Trust, Division of Gastroenterology, Manchester, UK; ${ }^{2}$ The Pennine Acute Hospitals NHS Trust, Department of Vascular and Endovascular Surgery, Manchester, UK; ${ }^{3}$ University of Maryland School of Medicine, Division of Gastroenterology and Hepatology, Baltimore, USA; ${ }^{4}$ Mayo Clinic, Division of Gastroenterology and Hepatology, Florida, USA; ${ }^{5}$ The Pennine Acute Hospitals NHS Trust, Division of Gastroenterology-Section of IBD, Manchester, UK
\end{abstract}

\subsection{6/gutjnl-2021-BSG.45}

Introduction The increasing incidence and prevalence of inflammatory bowel disease (IBD) has fuelled the need for innovative models of care. We aimed to compare effectiveness of e-health interventions with standard care in management of IBD.

Methods We searched Medline, Embase, PubMed, CINAHL, PsychInfo, Clinical trials registry and Cochrane databases for randomized controlled trials published in the English language until November 2020, comparing e-Health interventions to standard care for patients with IBD. Primary outcomes included difference in disease activity and patients in clinical remission at the end of follow up. Secondary outcomes included differences in quality of life (QoL), IBD-knowledge $\&$ rate ratios $(\mathrm{RR})$ for endoscopic procedures, total healthcare encounters, corticosteroid use, clinic visits and IBD related hospitalization or surgery. RevMan 5.4 was used for data analysis.

Results Nine studies ( $\mathrm{n}=1841$; 991- e-Health \& 850-controls) were identified. There was no statistically significant difference between the mean disease activity scores for ulcerative colitis (UC) [standard mean difference (SMD) 0.22 , 95\% confidence interval (CI): -0.04-0.48] and Crohn's disease (CD)(SMD 0.02, 95\% CI: -0.18-0.22) in the e-Health and standard care groups and no statistically significant difference in patients in clinical remission at the end of follow up between both groups (OR 1.05, 95\% CI: 0.76-1.45). Higher QoL (SMD 0.19, 95\% CI: $0.05-0.34$ ) and IBD knowledge (SMD 0.25, 95\% CI: $0.12-$ 0.37 ) scores were noted in the e-Health group compared to standard care. E-Health group had less clinic visits (RR 0.85, 95\% CI: $0.78-0.93$ ) while there were no statistically significant differences noted in the RR for endoscopic procedures, total healthcare encounters, corticosteroid use, and IBD-related hospitalization or surgery between both groups.

Conclusions E-Health interventions are comparable with standard care for impact on disease activity, remission, endoscopy utilization, total healthcare encounters, corticosteroid use, and IBD-related hospitalization or surgery. However, e-Health group had better outcomes with QoL, IBD related knowledge $\&$ fewer clinic visits.

\section{ATU-5 PERCUTANEOUS LIVER BIOPSY TO CONFIRM ADVANCED METASTATIC CANCER: A STEP TOO FAR?}

${ }^{1}$ Dominic King*, ${ }^{2}$ Benjamin Coupland, ${ }^{1}$ Anna Lock, ${ }^{3}$ Veronica Nanton, ${ }^{2}$ Prashant Patel, ${ }^{1}$ Nigel Trudgill. 'Sandwell and West Birmingham Hospitals NHS Trust, West Bromwich, UK; ${ }^{2}$ Informatics, University Hospitals Birmingham NHS Foundation Trust, Birimgham, UK;

${ }^{3}$ Social Science and Systems in Health, University of Warwick, Warwickshire, UK

\subsection{6/gutjnl-2021-BSG.46}

Introduction Liver biopsy carries significant risks, including bleeding and death. It is routinely undertaken to confirm imaging evidence of hepatic metastases. However, establishing a cancer diagnosis beyond doubt is of limited benefit if a patient is not suitable for oncological therapy. We have therefore examined outcomes in patients undergoing liver biopsy for metastatic cancer.

Methods Hospital Episode statistics were examined to identify patients undergoing percutaneous liver biopsy between 2010 and 2019 and diagnosed with metastatic cancer. Multivariable logistic regression examined risk factors for mortality at 14 and 30 days and receiving chemotherapy.

Results 30992 patients underwent liver biopsy for metastatic cancer (median age of 67 (IQR 59-74) years, 52\% female). $28 \%$ underwent biopsy during an emergency inpatient stay and $9 \%$ died within 14 days and $27.6 \%$ within 30 days of their biopsy. In contrast, only $2.2 \%$ of patients having an outpatient biopsy died within 14 days and $8.6 \%$ within 30 days.

Increased 30 day mortality was associated with: inpatient biopsy (odds ratio 3.37 (95\%CI 3.15-3.61)) and increasing comorbidity (Charlson score 1-4: 1.21 (1.11-1.32)). Lower 30 day mortality was associated with all ages under 70 (for 1829 yr olds: 0.35 (0.20-0.63)), a lymphoma diagnosis (0.69 (0.51-0.93)) and biopsy at a radiotherapy centre $(0.89$ (0.83$0.96))$.

$14,244(46 \%)$ patients received chemotherapy within 6 months of liver biopsy; 53\% of those undergoing outpatient biopsy but only $33 \%$ of those biopsied as an inpatient. $18 \%$ of patients received only one dose of chemotherapy and $18 \%$ died within 14 days of chemotherapy. Receiving chemotherapy was negatively associated with biopsy as an inpatient $(0.45$ (0.42-0.47)) and increasing comorbidity (Charlson score 1-4 0.85 (0.80-0.91)). All ages under 70 (for 18-29 yr olds: 3.79 (2.67-5.39)) and female sex (1.06 (1.01-1.11)) were associated with receiving chemotherapy. Medium and high volume providers of biopsies were also associated with receiving chemotherapy compared to the lowest volume providers (1.13 (1.041.22) and 1.51 (1.39-1.64), respectively).

Conclusions Mortality is high following liver biopsy to confirm metastatic cancer in patients admitted as an emergency. Only a third of such patients go on to receive chemotherapy. Clinicians should carefully consider the benefit of invasive 
diagnostics in those with metastatic cancer who are elderly, com-morbid or of poor performance status and multidisciplinary discussion including palliative care input may help such decision making.

\section{ATU-6 RESULTS OF RAPID HEPATITIS C TREATMENT SCALE-UP FOR PEOPLE WHO INJECT DRUGSIN TAYSIDE, SCOTLAND}

${ }^{1}$ Christopher Byrne* ${ }^{*}{ }^{1,2}$ John F Dillon. ${ }^{1}$ Molecular and Clinical Medicine, University of Dundee School of Medicine., Dundee, UK; ${ }^{2}$ Dept of Gastroenterology, Ninewells Hospital and Medical School, Dundee, UK

\subsection{6/gutjinl-2021-BSG.47}

In 2017, NHS Tayside rapidly scaled-up treatment among People Who Inject Drugs (PWID) for Hepatitis C (HCV) through novel care pathways. This was to drive the region towards HCV elimination by initiating treatment for 590 PWID and obtaining 540 Sustained Virologic Responses (SVR), over three years. In 2020, Tayside declared elimination of HCV. This study analyses: treatment outcomes for PWID treated from 2017-20 and the proportionate efficiency of treatment pathways.

Demographic and clinical parameters were collected for PWID treated for HCV using Direct Acting Antivirals (DAA) from January 2017-April 2020. Follow-up was censored December 2020. Anonymised data was stored. Descriptive statistics were performed using IBM SPSS 25. SVR was calculated as a proportion of treatments.

HCV treatment was initiated 713 times among 662 PWID. Patients were mostly male (74.2\%), and typically resided in deprived areas $(80.4 \%$ Scottish Index Multiple Deprivation 1 or 2). HCV genotype 3 was most common (56.5\%) followed by 1 (39.3\%). Liver cirrhosis was observed in a minority (9.1\%), and most reported receipt of Opiate Substitution Therapy (77.3\%). 573 (80.4\%) SVRs were observed. Excluding those with no SVR test $(n=89)$, this rises to $91.8 \%$. Highest SVR was observed in the community pharmacy pathway $(89.6 \%)$ but, excluding those without a test, the nurse-led community clinics yielded highest SVR at 96.2\%, followed by pharmacies at $95.6 \%$ (Table 1). Community pharmacies and the needle exchange pathway contributed almost half of all treatment initiations (49\%).

NHS Tayside exceeded its treatment target for the scale-up programme. The data demonstrate high levels of cure across novel pathways implemented in community settings. The data suggests moving HCV treatment from secondary to community care can significantly reduce the burden of $\mathrm{HCV}$ infection among PWID at a regional level.

\section{Posters - highly commended}

\section{Endoscopy}

\section{HTU-1 COMPUTER AIDED CHARACTERISATION OF COLORECTAL POLYPS USING ARTIFICIAL INTELLIGENCE}

\footnotetext{
1,2Rawen Kader*, ${ }^{3}$ Anton Mejias, ${ }^{3}$ Sanjith Hebbar, ${ }^{3}$ Patrick Brandao, 'Shahraz Islam, 1,2 Omer F Ahmad, ${ }^{2}$ Mohamed Hussein, ${ }^{4}$ Vinay Sehgal, ${ }^{4}$ Roser Vega, ${ }^{4}$ Edward Seward, ${ }^{3}$ Peter Mountney, ${ }^{1,2}$ Danail Stoyanov, ${ }^{1,2}$ LaurenceB Lovat. ${ }^{1}$ Wellcome/EPSRC Centre for Interventional and Surgical Sciences (WEISS), UCL, London, UK; ${ }^{2}$ Division of Surgery and Interventional Sciences, UCL, London, UK; ${ }^{3}$ Odin Vision, London, UK; ${ }^{4}$ University College London Hospital (UCLH), London, UK
}

\subsection{6/gutjil-2021-BSG.48}

Introduction Optical diagnosis is the in-vivo prediction of colorectal polyp histopathology. Inter-observer variability amongst endoscopists has limited its application in clinical practice. Artificial intelligence may lead to a new generation of clinical support tools capable of characterising polyps. Research in this field has often relied upon retrospective datasets, which are subject to sample selection bias, and consist of a limited number of images of each polyp.

Our aim was to develop a convolutional neural network (CNN) to characterise colorectal polyps as adenomatous or non-adenomatous using data collected prospectively.

Methods Video data was collected prospectively from colonoscopy procedures at a single centre using Olympus 260 and 290 series scopes. Histopathological classification, location and morphology was recorded for each polyp.

Video sequences of polyps in Narrow Band Imaging (NBI) and NBI-Near Focus (NBI-NF) were extracted. Both imaging modalities were used to increase the generalisability of the $\mathrm{CNN}$. Frames with poor visualisation of the polyp surface texture due to mucus, stool, halation or motion artifact were excluded. The ground truth for each frame was the polyp annotated with a bounding box and labelled with the histopathology.

A ResNet-101 CNN pre-trained on ImageNet was developed to classify the visual appearance of colorectal polyps as adenomatous or non-adenomatous.

Results The final dataset consisted of 371 histologically confirmed polyps (235 adenomas, 77 sessile serrated lesions, 58 hyperplastic, 1 traditional serrated adenoma) from 199 patients with a total of 31,110 video frames annotated. Data was split, as shown in Figure 1, into a training ( $\sim 50 \%)$, validation $(\sim 10 \%)$, and testing dataset $(\sim 40 \%)$ with no overlap of polyps or patients.

On a per-frame analysis, the accuracy of the CNN optical characterisation was 91\%, with a sensitivity of $91 \%$ to

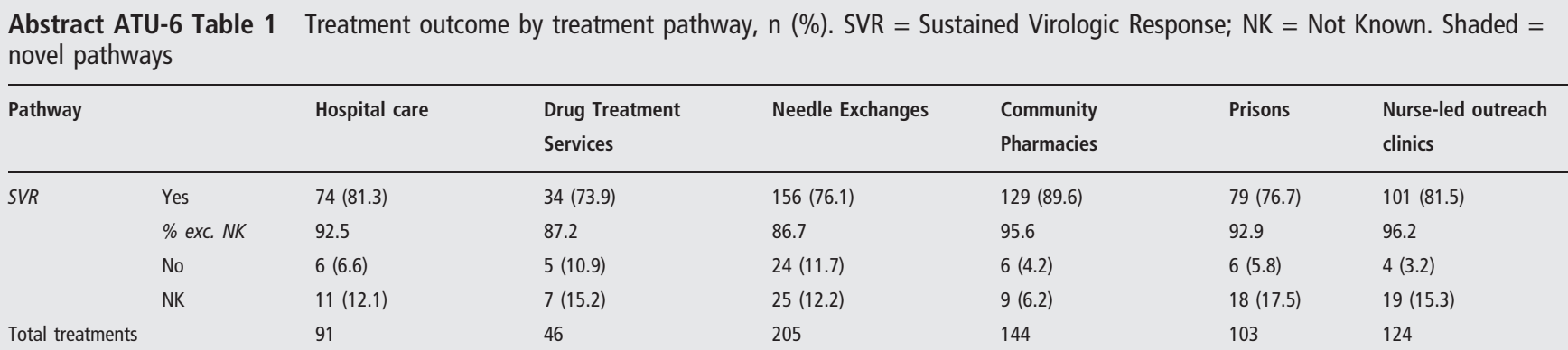

